\title{
Improving Effectiveness of a Coaching System Through Preference Learning
}

\author{
Martin Žnidaršič \\ Jožef Stefan Institute \\ Ljubljana, Slovenia \\ martin.znidarsic@ijs.si \\ Peter Rupnik \\ Jožef Stefan Institute \\ Ljubljana, Slovenia \\ peter.rupnik@ijs.si
}

\author{
Aljaž Osojnik \\ Jožef Stefan Institute \\ Ljubljana, Slovenia \\ aljaz.osojnik@ijs.si \\ Bernard Ženko \\ Jožef Stefan Institute \\ Ljubljana, Slovenia \\ bernard.zenko@ijs.si
}

\begin{abstract}
The paper describes an approach for indirect assessment and use of user preferences in an unobtrusive sensor-based coaching system with the aim of improving coaching effectiveness. The preference assessments are used to adapt the reasoning components of the coaching system in a way to better align with the preferences of its users. User preferences are learned based on data that describes user feedback as reported for different coaching messages that were received by the users. The preferences are not learned directly, but are assessed through a proxy - classifications or probabilities of positive feedback as assigned by a predictive machine learned model of user feedback. A brief description of the coaching setting is provided in the paper, before the approach for preference assessment is described and illustrated on a real-world example obtained during the testing of the coaching system with elderly users.
\end{abstract}

\section{CCS CONCEPTS}

\section{- Computing methodologies $\rightarrow$ Learning from critiques.}

\section{KEYWORDS}

Machine Learning, Preference Learning, User Centered Design

\section{ACM Reference Format:}

Martin Žnidaršič, Aljaž Osojnik, Peter Rupnik, and Bernard Ženko. 2021. Improving Effectiveness of a Coaching System Through Preference Learning. In The 14th PErvasive Technologies Related to Assistive Environments Conference (PETRA 2021), June 29-fuly 2, 2021, Corfu, Greece. ACM, New York, NY, USA, 7 pages. https://doi.org/10.1145/3453892.3461657

\section{INTRODUCTION}

Demographic and societal changes in recent decades are causing ever more people to live alone at older age. The usual tendency to live independently, coupled with supply of care facilities that is not coping with an increased demand, is fueling interest in ambient

This work is licensed under a Creative Commons

Attribution-NonCommercial-NoDerivs International 4.0 License.

PETRA 2021, June 29-fuly 2, 2021, Corfu, Greece

(c) 2021 Copyright held by the owner/author(s)

ACM ISBN 978-1-4503-8792-7/21/06.

https://doi.org/10.1145/3453892.3461657 assisted living (AAL) and sensor based monitoring and coaching solutions that would prolong the duration, ensure safety and improve the quality of independent living for the elderly.

An interesting system of this kind is the SAAM coaching system, which is being developed in scope of a collaborative research project with the same name (https://saam2020.eu/). Although it uses an array of sensor-based monitoring technologies, situation awareness reasoning engine and automated triggering of coaching actions, similarly as many other systems of this kind, the SAAM coaching system also tries to involve humans from the user's social circle in the coaching loop. Namely, the outputs of the system are not addressing only the user that is to be supported by the system, but in some situations also members of the user's social circle. For example, the system might not only directly suggest to the user that some physical activity would be advisable to keep-up with the user's usual levels of such an activity, but might opt to send a message to the user's friend or relative, and suggest them to invite the user for a walk together.

Besides trying to ensure unobtrusiveness of sensing and actuation, involving the user's social circle in coaching and having a friendly user interface, the system also tries to fit to the users with adaptation of some of the parameters of the coaching process according to user preferences. For this purpose, preference learning is used in order to gain information about the user preferences, which can then affect the parts of the system that can be adapted. Namely, although the reasoning models select suitable coaching actions based on the context as assessed by sensors, some situations have multiple suitable coachings and this leaves room for adaptation to individual users or groups of users. There are also some parameters related to rendering (form of presentation to the user) of the coachings, which are optional or even arbitrary, and these also can be made to suit the users and their preferences.

The aim of this paper is to describe a solution for assessment of user's preferences, its implementation and some experimental results. The solution is tailored to the specific coaching system, but the approach as such could be used also in many other similar systems. The contents of the paper is structured as follows: In Section 2 we present a selection of most relevant related work, in Section 3 we briefly describe the specific coaching system, which of its parameters we can adapt to the learned preferences and how the adaptation is done. Section4 describes the most important part of 
adaptation to preferences - the preference learning approach, supported by real-world examples. Concluding discussion and remarks are provided in Section 5.

\section{RELATED WORK}

Preferences are a concept present in many fields of science, ranging from economic decision theory [11] to AI [6]. They are especially important in multi-attribute decision theory, where they are commonly represented with value functions $[8,10]$. Given an attribute $X$ and $x_{i}$ as one of its possible values, value function $v\left(x_{i}\right)$ of the attribute's value $x_{i}$ models the value of $x_{i}$ for a decision maker and allows for modelling of relations to other possible attribute values, like whether they have equal value to the user, whether one is preferred over the other and by how much. The value function is commonly scaled to the interval $[0,1]$. Some examples of possible value functions are provided in Figure 1. The attributes that we are interested in (coaching action, persuasion strategy, interaction target) are nominal, like example (c) in Figure 1. Value function for such an attribute forms a distribution. Learning such a distribution in a given context is the goal of our approach, as we can use it in a distribution-based sampling procedure which ensures that the outputs of the coaching system are aligned with the user preferences.

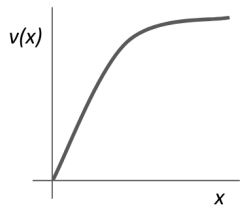

(a)

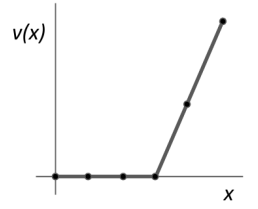

(b)

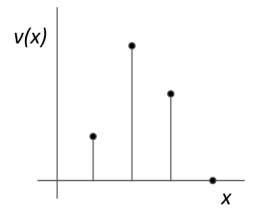

(c)
Figure 1: Examples of value functions: (a) a continuous value function that might hold, for example, for the value of one's salary; (b) value function for an ordinal attribute, for example, for the value of car's safety measured with NCAP points (in the shown example the user would be indifferent regarding values lower than 3); (c) value function in case of a nominal attribute such as color.

A value function can be estimated in a number of ways. In decision analysis it is usually estimated by questioning experts directly or indirectly with one of several possible approaches $[5,14]$. Evolutionary approaches, which are usually interactive are recently on the rise $[1,3]$, as are interactive machine-learning based approaches $[7,12,15]$. As we do not have access to the experts (users in our case) for the purpose of interactive preference assessment and we only have available indirect indications of preference, we use data-based modeling to approximate the value function of each of our attributes of interest in a given context. Of course, such an adaptive approach suffers from the cold start problem and can only be used once there is enough data available about the user's interaction with the system.

\section{ADAPTING COACHING TO USER'S PREFERENCES}

\subsection{The SAAM Coaching System}

The SAAM coaching system is a coaching system for elderly that aims to support its users to continue living independently. In contrast to typical AAL coaching approaches, it does not rely only on delivering coaching messages to elderly users directly, but primarily focuses on their social circles: family, friends, neighbors and organized caregivers. These are so-called secondary users (SUs), and are part of the coaching process: the system can address the coaching messages to them, and they can then act in the sense of a given coaching action. For example, the system might detect that lately the elderly that is being coached (a primary user (PU)) is not sleeping well, and issues the following coaching message to one or more of the person's SUs: "Instruct your PU to only go to bed if sleepy." The SU can then pay a visit to the PU or call him on the phone, ask about his sleeping quality, and suggest to go to bed only if really sleepy and not for other activities, like watching TV. The main idea of including user's social circles in the coaching process is not only to overcome the problem of elderly typically being averse to use modern technology, but also to improve their social activity. The above mentioned example falls in the domain of sleep quality coaching. In addition to this, SAAM targets three more domains: mobility, everyday activity (cooking, household, outside), and social activity.

The SAAM coaching system is quite complex and describing the details of its working is outside the scope of this paper. In the following paragraphs we only provide a high level description that should enable the reader to understand our preference learning approach and its integration with the coaching system. The basis of the SAAM coaching system are the so-called coaching pipelines $[4,17]$. For each of the coaching domains a separate coaching pipeline is implemented, and each consists of several components, as depicted in Figure 2. In essence, based on measurements from a set of ambiental and wearable sensors (smart electricity meter, inertial measurement unit (IMU) for sleep monitoring, sound detector, wearable IMUs, smartphone, etc.) a set of features is computed and these features are used as criteria or inputs for the next three components: situation assessment, coaching action modeling and coaching rendering modeling. Each of these components is realized as a multi-criteria decision-making (MCDM) model. As the name implies, the situation assessment model assesses the situation at a given point in time. For example, for sleep quality coaching, sleep is monitored with an IMU attached to bed, and from these measurements criteria such as sleep latency and sleep efficiency are assessed. These criteria are the inputs to the situation assessment model, and its output is an assessment whether the user slept well during the previous day or not. The coaching action model uses this information, combines it with the previously computed criteria and historical data, and proposes the most suitable coaching action. An example of a coaching action for the sleep quality domain is "Go to bed only if sleepy." With the coaching action identified we now need to decide how this action will be rendered, and this is the task of the coaching rendering model. Given a coaching action, this model again uses the computed criteria and historical data, as well as specific user's preferences as selected by the user in his profile. For example, some 
user might not want to share information about his sleep quality with his social circles, and decides to receive all sleep quality coaching directly and not through his SUs. In any case, the coaching rendering model aggregates all these data and outputs a specific rendering of a coaching action. For example, the above mentioned coaching action might be displayed to the PU directly using one of persuasion strategies, for example a suggestive persuasion strategy, resulting in a message "You might sleep better if you only go to bed if you feel sleepy." The actuation module takes care of presenting the message to the user, for example, by displaying it on the screen or as a voice message. In addition, the actuation module also enables us to collect user's feedback regarding each coaching action, which is done by pressing one of the buttons in the user interface: accept, cannot perform, or decline the coaching action. As we will see later, this is the basis of our preference learning approach described in Section 4.

The three above mentioned MCDM models are designed in collaboration with domain experts and are technically sets of hierarchically structured IF-THEN rules. These rules can produce one or more possible coaching actions (or its renderings), if the experts identified more than one suitable coaching action (rendering) for a given situation. Selection among more than one option can be done randomly using a suitable probability distribution. By default this distribution can be uniform, or it can be determined with preference learning, which is the topic of this paper.

\subsection{Preference Learning Targets}

In the previous subsection we have briefly described the inner working of our coaching system and saw that by adapting the probability distribution we can influence specific aspects of coaching. In our current setting we can influence three separate aspects, we call them preference learning targets (PLTS).

- Coaching action. Selecting a coaching action based on user's preference is only feasible in situations where several coaching actions are valid and appropriate. In essence, this requires that the coaching action decision model is probabilistic and at least a subset of possible coaching actions are selected stochastically according to some probability distribution. The probabilities from the preference learning module, described in Section 4, can be directly used in such a decision model.

- Persuasion strategy. SAAM coaching action messages are currently designed to follow two different persuasion strategies, suggestion and self monitoring. The hypothesis is that different persuasion strategies might be more effective for different people. Selection of the persuasion strategy is part of the coaching rendering model. At the start the uniform probability distribution is used, but extension with our preference learning module can be done in the same manner as for coaching actions.

- Interaction target (PU or SU). One of the main ideas of SAAM is that the coaching actions could be sent through primary user's social circle, that is, his or her secondary users. Given that people differ, some of the primary users might like this approach, while others might not appreciate it much. Therefore, this preference seems like a natural fit for personalization. Currently, each user can decide whether the coaching messages should be delivered to the user directly, through secondary users or both. If the user selected both, the interaction target can be selected randomly as in case of the other two PLTs above.

\subsection{Integration in the Coaching System}

Let us now present the whole process of preference learning and how it integrates in the coaching system. The process consists of the following steps.

- Collection of the learning data. At the beginning, when we start using the coaching system, we have no data that would contain information about user's preferences and that we could use for learning (cold start problem). Therefore we first need to collect such data, and we can do this, for example, by using the coaching system with preferences being selected randomly. Preferably, this random selection should follow a uniform probability distribution in order to cover the entire space of possible preferences, that is, the preferences that we aim to tailor in order to improve the acceptance of coaching actions. Such a uniformly distributed learning dataset also makes the model learning easier and typically the resulting model is also more accurate.

- Learning of the acceptance model. Once we have enough of the learning data collected we can learn a model that predicts user's acceptance of the received coaching actions as described in the next section. As we will see, this model can provide us with the assessment of the likelihood that each possible coaching action (with a selected rendering) will be accepted by the user. These probability estimates are then used in the next step.

- Using predictions of the acceptance model. These predicted probabilities can now be used to instantiate the probability distributions for each of the PLTs mentioned is Section 3.2. As a result, the coaching action and its rendering should now be adapted to the user's preference. We will illustrate how exactly this is done in Section 4.2.

The above three steps can be repeated during the deployment of the coaching system arbitrarily many times and in different settings. In general, we need to decide whose preferences we want to model, i.e., data from which users will be used for learning. These will probably also be the users for which the model will be used (although different settings are also possible, e.g., we might learn a model on a group of users and then use it on a new user that is similar to this group, etc.). In our case we might opt for learning preferences of all users, or preferences of individual users. Given a rather small amount of real-world data that we have available at the moment, our models presented in Section 4.3 will be learned on all users, but the procedure is the same in both cases.

\section{PREFERENCE LEARNING}

In this section we present our preference learning approach in more details. In fact, we do not learn the preferences or data instance ranking functions directly [9], but develop a single classification model for the user feedback (which can be valuable also for problem understanding and validation), and use its classifications or 


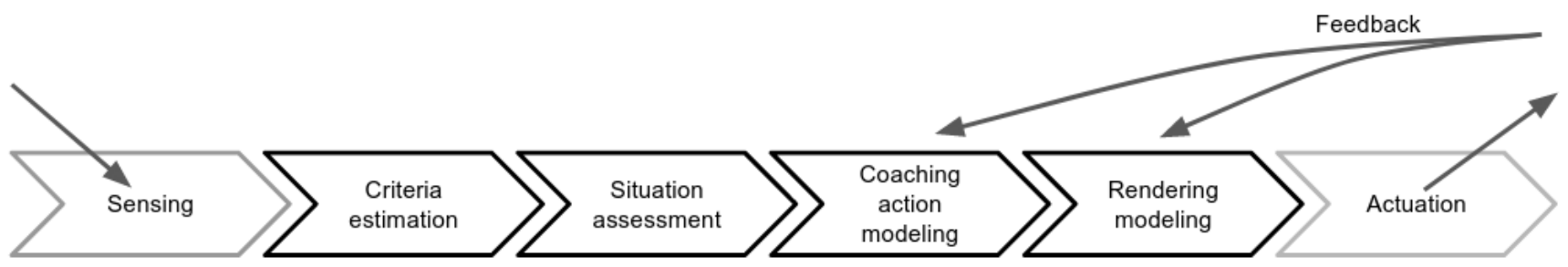

Figure 2: A diagram of a coaching pipeline, which is the basis of the SAAM coaching system.

probabilities of positive feedback as preference estimates. We will illustrate the procedure on a sleep quality coaching pipeline and we will address the three presented PLTs. However, the same procedure is applicable to other pipelines and PLTs in our coaching system as well.

\subsection{Learning Data}

Our aim is to learn about user's preferences and we can do this based on data comprising examples of different coaching actions issued with different renderings and user feedback reported in each of these cases. The data therefore needs to include attributes (or variables) describing: (I) the specific variable for the values of which we are interested in learning the user's preference (PLTs), (II) the context of the coaching action, and (III) the user feedback.

As explained in Section 3.2, PLTs are the coaching action, the persuasion strategy and the interaction target. The user feedback is the feedback provided about a specific coaching action that was issued. In SAAM, the feedback is provided by the users through a tablet application, where they can select to either accept, decline or cannot for each received coaching action. The context are all the other variables that are available to the preference learning system (information that we collect or compute about the situation, the user and the phenomena of interest), which might have an influence on the values of feedback. We can also include variables collected within pipelines covering other domains, if we think they might influence user's preferences.

We will illustrate our approach on data collected during the operation of our sleep quality coaching pipeline (the system is saving all the sensor measurements, computed features, model outputs and resulting coachings in the cloud). The data comprises 240 coaching actions addressed to 15 different users during a roughly three month period. In addition to the variables describing quality of sleep, we have also included some variables describing cooking activity that were collected in parallel during the operation of the cooking activity pipeline. Our motive for doing this was mostly to illustrate the possibility of including additional variables for describing context of a coaching action, although one could also assume that sleep quality is somewhat linked to cooking habits. All the attributes of our dataset are presented in Table 1, please note that we have merged the cannot and decline feedback into a single value.

\subsection{The Learning Approach}

Now that we have the learning data that is available for preference learning, we can start developing a model. Our idea is to learn a predictive model that is based on the input variables (PLTs and context) and predicts the user feedback, i.e., whether the user will accept (and hopefully fulfil) the issued coaching action, or not. We are therefore learning a predictive model $\mathrm{M}$ for the variable user feedback:

$$
\text { User Feedback }=M(\text { PLTs, } \text { Context }) \text {. }
$$

In principle we could use an arbitrary multi-variate learning or modelling approach, although we prefer approaches that provide, in addition to the predicted value, also some probability estimate for each of the possible outcomes. Such probability estimates make it easier to construct the probability distributions used in coaching activity and coaching rendering models.

A predictive model $M$ can be used to predict whether the user is likely to fulfil a specific received coaching action or not. How do we use such a model to assess the user's preferences for coaching? At a time when we have to issue a coaching action we have available all the values for the context variables, and we have to prepare one input instance for each possible PLT value and repeat this for all PLTs that we are addressing. Now we run all the instances generated in this way through the predictive model in order to collect predictions (and their probabilities) for the user feedback (coaching action acceptance). Based on these probabilities we can easily construct probability distributions required in coaching activity and coaching rendering models (Section 3.3).

Let us illustrate this procedure on an example. To make it simpler, let's address only two PLTs, persuasion strategy with possible values Suggestion and Self-monitoring, and interaction target with possible values $P U$ and $S U$. For this situation we can now construct four input instances:

$$
\begin{aligned}
& I_{1}=(\text { Per. Strategy }=\text { Suggestion }, \text { Int } . \text { Target }=\text { PU }, \text { Context }) \\
& I_{2}=(\text { Per. Strategy }=\text { Suggestion }, \text { Int } . \text { Target }=\text { SU }, \text { Context }), \\
& I_{3}=(\text { Per. Strategy }=\text { Self-monitoring }, \text { Int. Target }=\text { PU }, \text { Context }), \\
& I_{4}=(\text { Per. Strategy }=\text { Self-monitoring }, \text { Int } . \text { Target }=\text { SU, Context }) .
\end{aligned}
$$


Table 1: Attributes of the sleep quality coaching dataset, the dataset describes 240 coaching actions.

\begin{tabular}{lll}
\hline Attribute Name & Possible Values & Role \\
\hline Coaching Action & Get up from bed if awake, Go to bed only if sleepy, See your doctor, Adjust bedroom temperature, & PLT \\
& Avoid drinking 2h before sleep & PLT \\
Persuasion Strategy & Suggestion, Self-monitoring & PLT \\
Interaction Target & PU, SU & Context \\
Predicted Cooking Activity & None, Once, Twice or more & Context \\
Recorded Cooking Activity & None, Once, Twice or more & Context \\
Cooking Activity Situation & Usual cooking, Less cooking & Context \\
Subjective Sleep Quality & Numeric (0-3) & Context \\
Sleep Disturbance Type & None, Medical, Bathroom, Temperature & Context \\
Napping During Day & Yes, No & Context \\
Sleep Efficiency & Numeric & Context \\
Sleep Latency & Numeric & Target \\
User Feedback & Accept, Cannot or decline & \\
\hline
\end{tabular}

Running these four instances through a (hypothetical) model $M$ gives us four predictions for user feedback (acceptance) and probability estimates for user's acceptance $\left(P_{\text {acc }}\right)$ :

$$
\begin{aligned}
& M\left(I_{1}\right)=\text { Accept } P_{\text {acc }}\left(I_{1}\right)=0.75, \\
& M\left(I_{2}\right)=\text { Accept } ; P_{\text {acc }}\left(I_{2}\right)=0.65 . \\
& M\left(I_{3}\right)=\text { Decline } ; P_{\text {acc }}\left(I_{3}\right)=0.25, \\
& M\left(I_{4}\right)=\text { Decline } ; P_{\text {acc }}\left(I_{4}\right)=0.15 .
\end{aligned}
$$

Probability estimates obtained by most of machine learning methods are not true probabilities and are not normalized, but this is not a problem for our intended use. The above values can be transformed into distributions for both PLTs in a simple manner:

$$
\begin{aligned}
D(\text { Per. Strategy }) & =\left(\frac{P_{\mathrm{acc}}\left(I_{1}\right)+P_{\mathrm{acc}}\left(I_{2}\right)}{\sum_{i=1}^{4} P_{\mathrm{acc}}\left(I_{i}\right)}, \frac{P_{\mathrm{acc}}\left(I_{3}\right)+P_{\mathrm{acc}}\left(I_{4}\right)}{\sum_{i=1}^{4} P_{\mathrm{acc}}\left(I_{i}\right)}\right) \\
& =(0.78,0.22), \\
D(\text { Int. Target }) & =\left(\frac{P_{\mathrm{acc}}\left(I_{1}\right)+P_{\mathrm{acc}}\left(I_{3}\right)}{\sum_{i=1}^{4} P_{\mathrm{acc}}\left(I_{i}\right)}, \frac{P_{\mathrm{acc}}\left(I_{2}\right)+P_{\mathrm{acc}}\left(I_{4}\right)}{\sum_{i=1}^{4} P_{\mathrm{acc}}\left(I_{i}\right)}\right) \\
& =(0.56,0.44) .
\end{aligned}
$$

These two distributions can now be used directly in the coaching rendering model. As we can see, the adapted coaching rendering model will now much more likely use the suggestive persuasive strategy and will slightly prefer coaching to the PU.

\subsection{Preference Model for Sleep Quality Coaching}

As already mentioned, this approach works with any statistical or machine learning method that can learn the model $M$, however, for this illustrative example we decided to use simple decision trees and a random forest method. Both methods produce multivariate predictive models, but the first one gives us also an interpretable model that we will be able to inspect, and the second one also provides us with a ranking of the input variables. With the ranking we can somewhat understand how each variable, and in our case, also each PLT value influences the acceptance of the coaching action. All models that we present in the following subsection were learned with the Weka machine learning toolbox [16].
Let us now learn a decision tree model with the J48 algorithm [13]. Using the algorithm with the default parameters resulted in somewhat larger tree than can be presented here (with 24 nodes and 16 leaves), so we pruned it a bit only to see which variables appear at the root of the tree, since these tend to be the ones that influence the prediction most. Decision tree pruning is a standard procedure that removes the less informative attributes from the model in order to reduce complexity of a model, and usually results in a more robust model that generalizes the learning data better. The resulting tree is presented in Figure 3. Judging from the model, the variables that influence user acceptance are persuasion strategy used for the rendering of the coaching action, sleep efficiency, type of sleep disturbance and subjective sleep quality. The leaves of the tree (rectangles) contain the predicted value, a number of learning examples reaching that leaf and a number of misclassified examples by this leaf (the numbers are not integers because of missing values in the learning data, if the value of an attribute appearing in the split test of the tree is missing, the example is split to both branches). These values can also be used to assess the probability of a classification of a new example: we note which leaf was used for classification of a given example and assume that a leaf with less misclassifications provides predictions with higher reliability. Please note that since only one PLT (persuasion strategy) appears in the model, this model cannot be used to adapt probability distributions of the other two PLTs (coaching action and interaction target).

The second type of learning algorithm that we have applied to the same data is the random forest method. This is a well-known ensemble method [2] that is also based on decision trees, but as an ensemble method it combines multiple trees together and the final model is no longer interpretable. However, the method can also generate feature ranking scores for input variables and these scores can be used for estimating the influence that each of the variables has on the prediction. In our case the algorithm produces the scores presented in Table 2. The results confirm the importance of the variables identified by the decision tree (type of sleep disturbance, sleep efficiency and the persuasion strategy), although they suggest that other variables (subjective sleep quality and napping during 


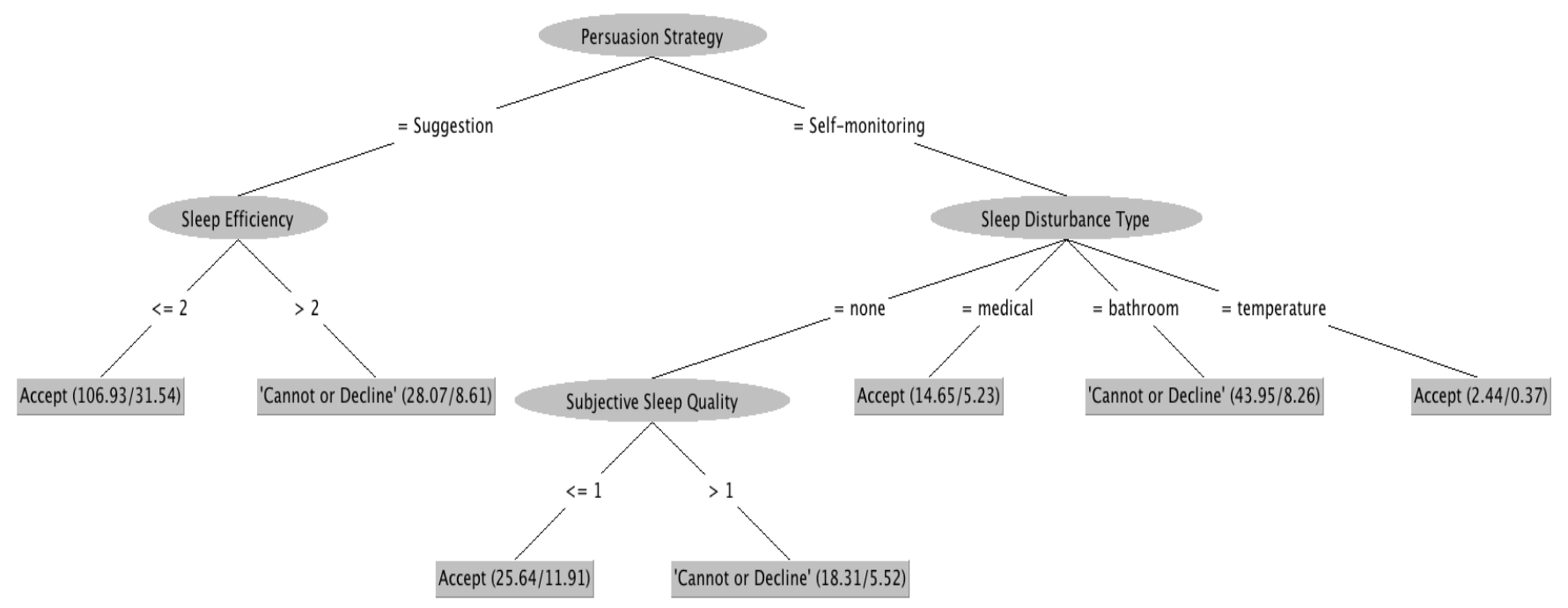

Figure 3: Decision tree for predicting user acceptance of a sleep quality coaching action.

Table 2: Ranking of the input variables with the random forest method for user acceptance of the sleep quality coaching actions. PLTs are typeset in bold.

\begin{tabular}{ll}
\hline Ranking Score & Input Variable \\
\hline 0.17 & Napping During Day \\
0.16 & Subjective Sleep Quality \\
0.16 & Sleep Disturbance Type \\
$\mathbf{0 . 1 4}$ & Persuasion Strategy \\
0.13 & Sleep Efficiency \\
0.11 & Sleep Latency \\
$\mathbf{0 . 1}$ & Coaching Action \\
0.06 & Predicted Cooking Activity \\
0.06 & Recorded Cooking Activity \\
$\mathbf{0 . 0 4}$ & Interaction Target \\
0.03 & Cooking Activity Situation \\
\hline
\end{tabular}

the day) are also important. If we only focus on our PLTs, we can see that the likelihood that the coaching action will be performed depends mostly on the persuasion strategy used when presenting the coaching action and the coaching action itself, and only slightly on the interaction target of this coaching action, i.e., whether the coaching message was sent to the primary or the secondary user. Of course, all these results need to be taken with a grain of salt and should not be used to draw any conclusions without further studies. They were obtained from a small data set and the distributions of some of the variables in it are quite skewed. As an example, upon a further investigation of the reason why the interaction target seems not to have any meaningful influence, we found that in the dataset the coaching actions addressed at secondary users are underrepresented.

Another point to discuss is the accuracy of these models. Using the 10 -fold cross-validation procedure, the presented decision tree model has a $68.3 \%$ accuracy (or $92.9 \%$ RRMSE - root relative squared error; here the value below $100 \%$ means that the model is better than chance) and the random forest model has a $75.4 \%$ accuracy (86.3\% RRMSE). These values are not stellar, but, in addition to the already mentioned small dataset, we need to be aware of two things. First, the problem that we are modelling is hard and the data that we can collect about this problem likely does not contain all information that we would need to reliably predict the preferences. Second, we do not use these predictions to construct the coaching models from scratch or to overrule the essential parts of the expertbased models, but we use them to improve the acceptance of the coaching actions within the specified flexibility. In this regard, if these models are better than chance, and we have shown that they are, they are already useful and can improve the results of coaching.

\section{CONCLUSIONS}

This paper describes the processes for assessment and integration of user preferences in a coaching system. It outlines the aspects of the coaching system that we can adapt according to user preferences, presents the preference learning approach and demonstrates it on a real-world data set. Although the results are preliminary, they confirm the feasibility and usefulness of the proposed approach.

There are several ways in which the learned preferences can be used to adapt the coaching system components and tailor them to the users. A selected procedure of this kind could also be integrated into the system in a way to allow automated adaptation. Although alluring, automation of a procedure that changes the reasoning components of the system can be harmful as it can cause the system to gradually change into a state in which it operates too differently than how it was envisioned. The adaptation approach can be made to be cautious (e.g., not to be able to change zero probability coaching actions to non-zero ones), but since coaching affects people and since the coaching systems and coaching feedback and not yet fully studied and understood phenomena, we think that regardless of the execution mode, manual or automated, the adaptations to user 
preferences should be monitored by the system operators and domain experts and not left to be changed according to data without human oversight.

\section{ACKNOWLEDGMENTS}

We wish to acknowledge funding from the $\mathrm{EU}$ via the $\mathrm{H} 2020$ project SAAM (Grant No. 769661) and from the Slovenian Research Agency through research core funding of the program P2-0103.

\section{REFERENCES}

[1] Jürgen Branke, Salvatore Greco, Roman Słowiński, and Piotr Zielniewicz. 2014 Learning value functions in interactive evolutionary multiobjective optimization. IEEE Transactions on Evolutionary Computation 19, 1 (2014), 88-102.

[2] Leo Breiman. 2001. Random Forests. Machine Learning 45, 1 (2001), 5-32.

[3] Laura Cruz-Reyes, Eduardo Fernandez, and Nelson Rangel-Valdez. 2017. A metaheuristic optimization-based indirect elicitation of preference parameters for solving many-objective problems. International fournal of Computational Intelligence Systems 10, 1 (2017), 56-77.

[4] Yordan Dimitrov, Zlatka Gospodinova, Richard Wheeler, Martin Žnidaršič, Bernard Ženko, Vera Veleva, and Nadejda Miteva. 2019. Social Activity Modelling and Multimodal Coaching for Active Aging. In Proceedings of the 12th ACM International Conference on PErvasive Technologies Related to Assistive Environments (Rhodes, Greece) (PETRA '19). Association for Computing Machinery, New York, NY, USA, 608-615.

[5] Peter H. Farquhar and Robin L. Keller. 1989. Preference intensity measurement. Annals of operations research 19, 1 (1989), 205-217.

[6] Johannes Fürnkranz and Eyke Hüllermeier (Eds.). 2010. Preference Learning. Springer, Berlin.
[7] Johannes Fürnkranz and Eyke Hüllermeier. 2010. Preference Learning and Ranking by Pairwise Comparison. In Preference Learning, Johannes Fürnkranz and Eyke Hüllermeier (Eds.). Springer, Berlin, 65-82.

[8] Salvatore Greco, Jose Figueira, and Matthias Ehrgott. 2016. Multiple criteria decision analysis (2nd ed.). Springer, New York.

[9] Eyke Hüllermeier and Johannes Fürnkranz. 2013. Preference learning and ranking. Machine Learning 93, 2 (2013), 185-189.

[10] Ralph L. Keeney, Howard Raiffa, and Richard F. Meyer. 1993. Decisions with multiple objectives: preferences and value trade-offs. Cambridge university press, Cambridge.

[11] R. Duncan Luce and Howard Raiffa. 1957. Games and Decisions: Introduction and Critical Survey. Wiley, New York.

[12] Giovanni Misitano. 2020. Interactively Learning the Preferences of a Decision Maker in Multi-objective Optimization Utilizing Belief-rules. In 2020 IEEE Symposium Series on Computational Intelligence (SSCI). IEEE, New York, 133-140.

[13] Ross J. Quinlan. 1993. C4.5: programs for machine learning. Morgan Kaufmann Publishers Inc., San Francisco, CA, USA.

[14] Ahti A. Salo and Raimo P. Hamalainen. 2001. Preference ratios in multiattribute evaluation (PRIME)-elicitation and decision procedures under incomplete information. IEEE Transactions on Systems, Man, and Cybernetics-Part A: Systems and Humans 31, 6 (2001), 533-545.

[15] David S. Todd and Pratyush Sen. 1999. Directed multiple objective search of design spaces using genetic algorithms and neural networks. In Proceedings of the 1st Annual Conference on Genetic and Evolutionary Computation-Volume 2. Morgan Kaufmann, San Francisco, 1738-1743.

[16] Ian H. Witten, Eibe Frank, Mark A. Hall, and Chris J. Pal. 2016. Data Mining: Practical Machine Learning Tools and Techniques. Elsevier Science, Amsterdam.

[17] Martin Žnidaršič, Bernard Ženko, Aljaž Osojnik, Marko Bohanec, Panče Panov, Helena Burger, Zlatko Matjačić, and Mojca Debeljak. 2019. Multi-criteria Modelling Approach for Ambient Assisted Coaching of Senior Adults. In Proceedings of the 11th International foint Conference on Knowledge Discovery, Knowledge Engineering and Knowledge Management - Volume 2: KEOD. INSTICC, SciTePress, Vienna, 87-93. 